\title{
A HIERARCHICAL ALGORITHM FOR CAUSALITY DISCOVERY AMONG ATRIAL FIBRILLATION ELECTROGRAMS
}

\author{
David Luengo , Gonzalo Ríos-Muñoz , Víctor Elvira , Antonio Artés-Rodríguez \\ Univ. Politécnica de Madrid, Ctra de Valencia, Km. 7, 28031 Madrid (Spain) \\ Univ. Carlos III de Madrid, Av. de la Universidad, 30, 28911 Leganés (Spain) \\ Gregorio Marañón Health Research Institute, Dr. Esquerdo 46, Madrid (Spain)
}

\begin{abstract}
Multi-channel intracardiac electrocardiograms (electrograms) are sequentially acquired, at the electrophysiology laboratory, in order to guide radio frequency catheter ablation during heart surgery performed on patients with sustained atrial fibrillation (AF). These electrograms are used by cardiologists to determine candidate areas for ablation (e.g., areas corresponding to high dominant frequencies or complex fractionated electrograms). In this paper, we introduce a novel hierarchical algorithm for causality discovery among these multi-output sequentially acquired electrograms. The causal model obtained provides important information about the propagation of the electrical signals inside the heart, uncovering wavefronts and activation patterns that will serve to increase our knowledge about $\mathrm{AF}$ and guide cardiologists towards candidate areas for catheter ablation. Numerical results on synthetic signals, generated using the FitzHugh-Nagumo model, show the good performance of the proposed approach.
\end{abstract}

Index Terms - electrocardiography, atrial fibrillation, Granger causality

\section{INTRODUCTION}

Atrial fibrillation (AF), which is a family of cardiac diseases characterized by a rapid and unsynchronized contraction of the atria, is the most common cardiac arrhythmia. Indeed, AF has reached epidemic proportions [1], with one out of four people over 40 years old predicted to suffer from $\mathrm{AF}$ in the future [2]. However, its underlying mechanisms are still not fully understood, and several theories for the initiation and maintenance of $\mathrm{AF}$ have been proposed $[3,4,5]$. One of the leading hypotheses (rotor theory) states that specific areas of the myocardium are responsible for $\mathrm{AF}$ initiation and maintenance. RF catheter ablation, where an RF catheter placed inside the heart is used to ablate the areas causing AF, is increas-

This work has been supported by the Spanish government's projects DISSECT (TEC2012-38058-C03-01), ALCIT (TEC2012-38800-C03-01), OTOSiS (TEC2013-41718-R), and COMPREHENSION (TEC2012-38883C02-01). D. Luengo has also been funded by "I Convocatoria de Ayudas Fundación BBVA a Investigadores, Innovadores y Creadores Culturales". ingly used, but requires identifying arrhythmogenetic areas. Sites with high dominant frequencies or complex fractionated electrograms have been proposed as candidates for ablation, but the performance of these approaches is still unsatisfactory.

Several authors have investigated the inference of causality relationships among different biomedical signals [6, 7]. In particular, causality discovery tools have been extensively used in neurology [8]. In cardiology, on the one hand Granger causality has been used to investigate the relationship between several physiological time series $[9,10]$. On the other hand, the use of partial directed coherence to investigate propagation patterns in intra-cardiac signals was considered in [11], whereas Granger causality maps were built in $[12,13]$. However, all of these approaches are based on the standard full conditional approach to causality discovery.

In this paper, we propose a novel hierarchical causality approach for causality discovery among AF electrograms. The algorithm initially selects the node with the highest number of potential causal links as the root node. The candidate sons of the root node are then explored (starting by the closest node), accepting them as true sons only if they provide relevant information conditioned on all previously accepted sons. This process is repeated until there are no more nodes left to process. The causality maps inferred contain important information about the propagation of the electrical signals inside the heart, allowing us to uncover wavefronts and activation patterns that will serve to increase our knowledge about $\mathrm{AF}$ and guide cardiologists towards candidate areas for ablation.

The paper is organized as follows. Firstly, Section 2 introduces Granger causality, describing both the pairwise and conditional approaches. Then, Section 3 details the proposed hierarchical causality algorithm, Section 4 shows the numerical results, and Section 5 provides the conclusions.

\section{GRANGER CAUSALITY (G-CAUSALITY)}

Let us assume that we have $N$ samples of a multi-variate time series composed of $Q$ signals, $x_{q}[n]$ for $q=1, \ldots, Q$ and $n=0,1, \ldots, N-1$, which correspond to unipolar intracardiac ECGs (electrograms) recorded at a single heart site 
during RF catheter ablation therapy. In the sequel, we describe two standard approaches for causality inference.

\subsection{Standard Pairwise Causality}

In its standard (pairwise) formulation, Granger causality (Gcausality) measures the increase in predictability on the future outcome of a given signal, $x_{q}[n]$ with $1 \leq q \leq Q$, given the past values of another signal, $x_{\ell}[n]$ with $1 \leq \ell \leq Q$, w.r.t. the predictability achieved by taking into account only past values of $x_{q}[n]$ [14]. The linear autoregressive (AR) predictor for $x_{q}[n]$ given its past samples (i.e., the $q$-th self-predictor) is given by

$$
\hat{x}_{q}[n]=\sum_{m=1}^{M_{q q}} \alpha_{q q}[m] x_{q}[n-m]=\boldsymbol{\alpha}_{q q}^{\top} \mathbf{x}_{q}[n],
$$

where $M_{q q}$ is the order of the predictor (obtained typically using some penalization for model complexity to avoid overfitting [15]), $\alpha_{q q}[m]$ are the coefficients of the model, $\boldsymbol{\alpha}_{q q}=\left[\alpha_{q q}[1], \ldots, \alpha_{q q}\left[M_{q q}\right]\right]^{\top}$, and $\mathbf{x}_{q}[n]=$ $\left[x_{q}[n-1], \ldots, x_{q}\left[n-M_{q q}\right]\right]^{\top}$. Similarly, let us define the linear AR predictor for $x_{q}[n]$ given the past samples of both $x_{q}[n]$ and $x_{\ell}[n]$ (i.e., the cross-predictor from the $\ell$-th signal to the $q$-th signal) as

$$
\hat{x}_{\ell \rightarrow q}[n]=\boldsymbol{\alpha}_{q q}^{\top} \mathbf{x}_{q}[n]+\boldsymbol{\alpha}_{\ell q}^{\top} \mathbf{x}_{\ell}[n],
$$

where $M_{\ell q}$ is the order of the predictor from the $\ell$-th signal to the $q$-th output (different from $M_{q q}$ in general), $\alpha_{\ell q}[m]$ its coefficients, $\alpha_{\ell q}=\left[\alpha_{\ell q}[1], \ldots, \alpha_{\ell q}\left[M_{\ell q}\right]\right]^{\top}$ and $\mathbf{x}_{\ell}[n]=$ $\left[x_{\ell}[n-1], \ldots, x_{\ell}\left[n-M_{\ell q}\right]\right]^{\top}$. The residual errors of the two predictors in (1) and (2) are $\varepsilon_{q}[n]=x_{q}[n]-\hat{x}_{q}[n]$ and $\varepsilon_{\ell \rightarrow q}[n]=x_{q}[n]-\hat{x}_{\ell \rightarrow q}[n]$ respectively. The pairwise $G$ causality strength is then measured by the logarithm of the ratio of these two variances [16]:

$$
G_{\ell \rightarrow q}=\ln \frac{\operatorname{Var}\left(\varepsilon_{q}[n]\right)}{\operatorname{Var}\left(\varepsilon_{\ell \rightarrow q}[n]\right)} .
$$

Using these pairwise values, we can build a pairwise $G$ causality strength matrix, $\mathrm{G}$, whose $(\ell, q)$-th entry is ${ }^{1}$

$$
\mathbf{G}_{\ell, q}= \begin{cases}G_{\ell \rightarrow q}, & \ell \neq q ; \\ 0, & \ell=q .\end{cases}
$$

Finally, note that we should add a causality link from $\ell$ to $q$ only when the decrease in the residual's noise variance from (1) to (2) is statistically significant. In order to construct this causality graph, we may define the pairwise $G$-causality connection matrix, $\mathbf{C}$, whose $(\ell, q)$-th element is $C_{\ell \rightarrow q}=\mathbb{I}_{p}\left(G_{\ell \rightarrow q}\right)$, where $\mathbb{I}_{p}(\cdot)$ is an indicator function such that $\mathbb{I}_{p}\left(G_{\ell \rightarrow q}\right)=1$ when the causal link from $\ell$ to $q$ is statistically significant (as indicated by its $p$-value for example) and $\mathbb{I}_{p}\left(G_{\ell \rightarrow q}\right)=0$ otherwise.

\footnotetext{
${ }^{1}$ Note that $\operatorname{Var}\left(\varepsilon_{q \rightarrow q}[n]\right)=\operatorname{Var}\left(\varepsilon_{q}[n]\right)$, since $\hat{x}_{q \rightarrow q}[n]=\hat{x}_{q}[n]$, so $G_{q \rightarrow q}[n]=\ln 1=0$ and the definition in (4) is consistent with (3).
}

\subsection{Conditional G-Causality}

Pairwise causality is unable to discriminate between direct causal relationships (i.e., between parents and sons) and indirect relationships (e.g., between grandparents and grandchildren). In order to avoid the undesired extra edges introduced by these indirect relationships, [16] proposed the use of conditional $G$-causality. Let us define as $\mathcal{I}$ the set containing the indexes of the conditioning variables. Now we can define the conditional self-predictor as

$$
\hat{x}_{q \mid \mathcal{I}}[n]=\boldsymbol{\alpha}_{q q}^{\top} \mathbf{x}_{q}[n]+\sum_{r \in \mathcal{I}} \boldsymbol{\alpha}_{r q}^{\top} \mathbf{x}_{r}[n],
$$

where $\alpha_{r q}=\left[\alpha_{r q}[1], \ldots, \alpha_{r q}\left[M_{r q}\right]\right]^{\top}$ and $\mathrm{x}_{r}[n]=\left[x_{r}[n-\right.$ $\left.1], \ldots, x_{r}\left[n-M_{r q}\right]\right]^{\top}$ for all $r \in \mathcal{I}$, and the conditional cross-predictor from the $\ell$-th signal (with $\ell \notin \mathcal{I}$ ) to the $q$-th output as

$$
\hat{x}_{\ell \rightarrow q \mid \mathcal{I}}[n]=\boldsymbol{\alpha}_{q q}^{\top} \mathbf{x}_{q}[n]+\sum_{r \in \mathcal{I}} \boldsymbol{\alpha}_{r q}^{\top} \mathbf{x}_{r}[n]+\boldsymbol{\alpha}_{\ell q}^{\top} \mathbf{x}_{\ell}[n] .
$$

Now, by defining the residual errors as $\varepsilon_{q \mid \mathcal{I}}[n]=x_{q}[n]-$ $\hat{x}_{q \mid \mathcal{I}}[n]$ and $\varepsilon_{\ell \rightarrow q \mid \mathcal{I}}[n]=x_{q}[n]-\hat{x}_{\ell \rightarrow q \mid \mathcal{I}}[n]$, the conditional G-causality strength can be defined as

$$
G_{\ell \rightarrow q \mid \mathcal{I}}=\ln \frac{\operatorname{Var}\left(\varepsilon_{q \mid \mathcal{I}}[n]\right)}{\operatorname{Var}\left(\varepsilon_{\ell \rightarrow q \mid \mathcal{I}}[n]\right)} .
$$

Just like in the case of the pairwise causality, we may define two conditional connection/strength G-causality matrices, $\mathbf{G}_{\mathcal{I}}$ and $\mathbf{C}_{\mathcal{I}}$, whose $(\ell, q)$-th elements are respectively $\mathbf{G}_{\ell, q \mid \mathcal{I}}=G_{\ell \rightarrow q \mid \mathcal{I}}$ and $C_{\ell \rightarrow q \mid \mathcal{I}}=\mathbb{I}_{p}\left(G_{\ell \rightarrow q \mid \mathcal{I}}\right)^{2}$

\section{HIERARCHICAL GRANGER CAUSALITY}

On the one hand, pairwise G-causality may provide misleading results, as it includes both direct and indirect causal relationships. On the other hand, the "brute-force approach" to conditional causality (i.e., applying conditional causality on the whole data set all at once) is much more demanding from a computational point of view and may obscure some of the existing relationships. Hence, in this paper we propose a hierarchical approach that is able to exploit the advantages of both approaches while minimizing their drawbacks. The algorithm starts by searching for the node with the highest number of Gcausality links to the other nodes and selecting it as the root node. Then, the sons of the root node are processed sequentially according to their proximity, adding new causality links if they are significant conditioned on the previously added

\footnotetext{
${ }^{2}$ Note that the pairwise G-causality connection/strength matrices are unique, whereas many conditional G-causality connection/strength matrices can be constructed. The most usual situation in the literature is setting $\mathcal{I}=\mathcal{S}_{\neg \ell}=\{1, \ldots, \ell-1, \ell+1, \ldots, Q\}=\{1, \ldots, Q\} \backslash\{\ell\}$ and constructing the full conditional $\mathrm{G}$-causality connection/strength matrices as $\mathbf{G}_{\ell, q \mid S_{\neg \ell}}=G_{\ell \rightarrow q \mid S_{\neg \ell}}$ and $C_{\ell \rightarrow q \mid S_{\neg \ell}}=\mathbb{I}_{p}\left(G_{\ell \rightarrow q \mid S_{\neg \ell}}\right)$ respectively.
} 
links. This process is repeated iteratively (on the grandsons of the root node and so on) until there are no more nodes to process and a poly-tree has been constructed. The following two assumptions are made in building this poly-tree:

1. No feedback links can exist from lower nodes to higher nodes in the hierarchy.

2. The causal interaction is given by neighbouring nodes.

In the sequel, we describe the steps of the algorithm in detail.

\subsection{Initialization: Selecting the Root Node}

The initialization stage seeks to find the optimal root node for the causal graph. This is done by calculating the pairwise G-causality among all nodes and selecting the one with the highest number of G-causality links to the other nodes. As a result, this stage returns the root node, $i_{1}$, and the set of its candidate sons, $\mathcal{C}_{1}=\operatorname{cand}\left\{i_{1}\right\}$. The detailed steps taken are:

1. Set $\mathbf{G}=\mathbf{0}$ and $\mathbf{C}=\mathbf{0}$. Initialize the sets of sons and parents as empty sets: $\mathcal{P}_{q}=\operatorname{pa}\{q\}=\emptyset$ and $\mathcal{S}_{q}=$ $\operatorname{son}\{q\}=\emptyset$ for $q=1, \ldots, Q$.

2. $F O R q=1, \ldots, Q-1$ and $\ell=q+1, \ldots, Q$ : Calculate $G_{q \rightarrow \ell}$ and $G_{\ell \rightarrow q}$, and set the corresponding entries in $\mathrm{G}$ and $\mathrm{C}$.

3. Calculate the G-causality strength of the $q$-th node ( $q=1, \ldots, Q-1$ ) as the sum of the strength of its causal links to the remaining nodes, $g_{q}=$ $\sum_{\ell=1}^{Q} \mathbf{G}_{q, \ell}=\sum_{\ell=1}^{Q} G_{q \rightarrow \ell}$, and the number of links for each node as $K_{q}=\sum_{\ell=1}^{Q} \mathbf{C}_{q, \ell}=\sum_{\ell=1}^{Q} \mathbb{I}_{p}\left(G_{q \rightarrow \ell}\right)$.

4. Determine the node with the highest number of causal links stemming from it,

$$
i_{1}=\underset{1 \leq q \leq Q}{\arg \max } K_{q}
$$

and set it as the root node, with $g_{q}$ being used only to discriminate among nodes with identical values of $K_{q}$.

5. Obtain the set of candidate sons of the root node: $\mathcal{C}_{i_{1}}=$ cand $\left\{i_{1}\right\}=\left\{\ell: \mathbf{C}_{i_{1}, \ell}=1\right\}$.

\subsection{First Iteration: Processing the Sons of the Root Node}

This stage is in charge of processing the set of candidate sons of the root node, determining which of them are true sons. This decision is taken by sorting the candidates according to their proximity to the root node, and processing them sequentially (with closer candidates being processed first). At each iteration, a conditional G-causality strength is calculated using the current set of sons of the root node (initially empty). If the G-causality connection is deemed statistically significant (by means of its $p$-value), the candidate is added to the set of sons of the root node and the corresponding entry in the conditional G-causality connectivity/strength matrices is updated. The motivation for this approach is that true sons still provide statistically significant G-causality values after conditioning, whereas descendants further away along the family tree do not provide statistically significant $G$-causality values (as they are masked by closer descendants of the root node). As a result, this stage sets the corresponding entries in the strength/connection G-causality matrices, $\mathbf{G}_{\ell, q \mid \mathcal{P}}$ and $\mathbf{C}_{\ell, q \mid \mathcal{P}}$, returns the set of sons of the root node, $\mathcal{S}_{i_{1}}=\operatorname{son}\left\{i_{1}\right\}$, and sets the root node as the parent for the nodes in $\mathcal{S}_{i_{1}}$, i.e., $\mathcal{P}_{q}=\operatorname{pa}\{q\}=\left\{i_{1}\right\} \forall q \in \mathcal{S}_{i_{1}}$. The procedure applied is:

1. Set $\mathbf{G}_{i_{1}, q \mid P}=0$ and $\mathbf{C}_{i_{1}, q \mid P}=0$ for $q=1, \ldots, Q$. Set $\mathbf{G}_{\ell, q \mid \mathcal{P}}=\mathrm{NaN}$ and $\mathbf{C}_{\ell, q \mid \mathcal{P}}=\mathrm{NaN}$ for $1 \leq \ell, q \leq Q$ with $\ell \neq i_{1}{ }^{3}$

2. Sort the elements in $\mathcal{C}_{i_{1}}$ according to their proximity to the root node, with the distance defined as:

$d\left(i_{1}, j\right)=\min \left\{\left(\left(i_{1}-j\right)\right)_{Q},\left(\left(j-i_{1}\right)\right)_{Q}\right\}, \quad \forall j \in \mathcal{C}_{i_{1}}$,

with $((k))_{Q}$ denoting the modulo operation, i.e., for any three integer numbers $m, k$ and $Q, m=((k))_{Q} \Leftrightarrow$ $k=\ell Q+m$, where $\ell$ and $m$ are the only integers such that $-\infty<\ell<\infty$ and $0 \leq m \leq Q-1$.

3. Set $C_{i_{1} \rightarrow C_{i_{1}}(1) \mid \mathcal{P}}=1, G_{i_{1} \rightarrow C_{i_{1}}(1) \mid \mathcal{P}}=G_{i_{1} \rightarrow C_{i_{1}}(1)}$ and $\mathcal{S}_{i_{1}}=\operatorname{son}\left\{i_{1}\right\}=\left\{\mathcal{C}_{i_{1}}(1)\right\}$.

4. $\operatorname{FOR} j=2, \ldots,\left|\mathcal{C}_{i_{1}}\right|$ :

(a) Calculate $G_{i_{1} \rightarrow C_{i_{1}}(j) \mid \mathcal{S}_{i_{1}}}$ and $C_{i_{1} \rightarrow C_{i_{1}}(j) \mid \mathcal{S}_{i_{1}}}=$ $\mathbb{I}_{p}\left(G_{i_{1} \rightarrow c_{i_{1}}(j) \mid S_{i_{1}}}\right)$.

(b) IF $C_{i_{1} \rightarrow c_{i_{1}}(j) \mid \mathcal{S}_{i_{1}}}=1$ : Set $C_{i_{1} \rightarrow \mathcal{C}_{i_{1}}}(j) \mid P=1$, $G_{i_{1} \rightarrow C_{i_{1}}(j) \mid \mathcal{P}}=G_{i_{1} \rightarrow \mathcal{C}_{i_{1}}(j)}, \mathcal{S}_{i_{1}}=\mathcal{S}_{i_{1}} \cup\left\{\mathcal{C}_{i_{1}}(j)\right\}$ and $\mathcal{P}_{C_{i_{1}}(j)}=\operatorname{pa}\left\{\mathcal{C}_{i_{1}}(j)\right\}=\left\{i_{1}\right\}$.

\subsection{Main Algorithm: Processing the Remaining Nodes Iteratively}

This final stage is in charge of processing the remaining roots iteratively in a hierarchical fashion. The process described in the previous section is repeated iteratively, processing the sons of each of the sons of the root node (i.e., the grandsons of the root node), starting again by the closest one. The algorithm proceeds in this way (i.e., processing the greatgrandsons of the root node, the great-great-grandsons of the root node and so on), until there are no more nodes to process. This stage returns the full strength/connection G-causality matrices, $\mathbf{G}_{\ell, q \mid \mathcal{P}}$ and $\mathbf{C}_{\ell, q \mid \mathcal{P}}$, defining a causal network with the corresponding sets of sons and parents, $\mathcal{S}_{q}=\operatorname{son}\{q\}$ and $\mathcal{P}_{q}=\operatorname{pa}\{q\}$ for $q=1, \ldots, Q$. The steps taken are:

${ }^{3}$ NaN is the IEEE arithmetic representation for "Not-a-Number", which is obtained as the result of mathematically undefined operations (e.g., $0 / 0$ or $\infty-\infty$ ). We use it here as a convenient way to indicate entries of $\mathbf{G}_{p}$ and $\mathbf{C}_{\mathcal{P}}$ that have not been defined yet. 

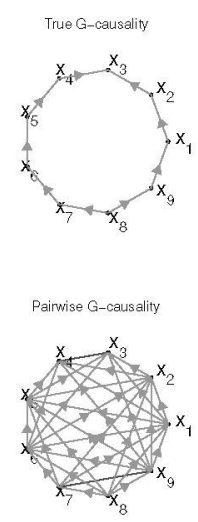
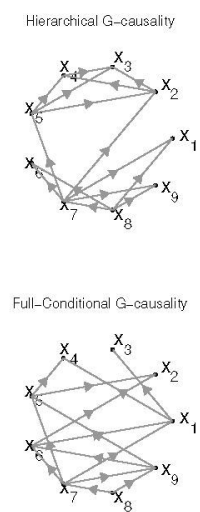

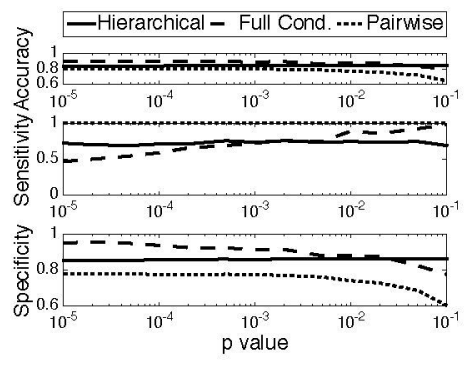

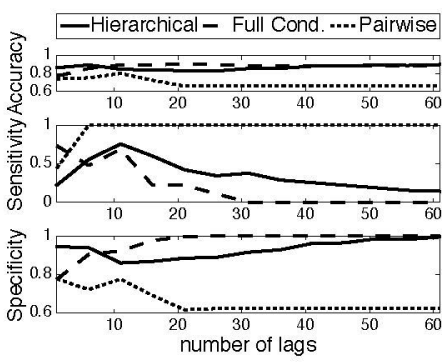

Fig. 1. (left) True map and causality maps inferred $(M=11, p=0.01$ and $\mathrm{SNR}=30 \mathrm{~dB})$. (center) Effect of changing the $p$-value $(M=11$ and SNR=30 dB). (right) Effect of changing the number of lags $M(p=0.01$ and $\mathrm{SNR}=30 \mathrm{~dB})$.

1. Set $t=1, \mathcal{I}_{t}=\left\{i_{1}\right\}$ and $\mathcal{M}_{t}=\{1, \ldots, Q\} \backslash \mathcal{I}_{t}$.

2. WHILE $\mathcal{M}_{t} \neq \emptyset$ :

(a) $\operatorname{FOR} k=1, \ldots,\left|\mathcal{I}_{t}\right|$ :

- Set $\mathcal{S}=\mathcal{S}_{\mathcal{I}_{t}(k)}$ and sort its elements according to their proximity to the current parent.

- $\mathrm{FOR} \ell=1, \ldots,|\mathcal{S}|$ and $j=1, \ldots,|\mathcal{C}|$ : Set $\mathcal{C}=\mathcal{C}_{S(\ell)}=\mathcal{M}_{t} \backslash \mathcal{S}(\ell)$, and calculate $G_{S(\ell) \rightarrow C(j) \mid I_{t}}$ and $C_{S(\ell) \rightarrow \mathcal{C}(j) \mid \mathcal{I}_{t}}=$

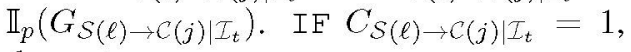
then set:

$$
\begin{aligned}
C_{\mathcal{S}(\ell) \rightarrow C(j) \mid \mathcal{P}} & =1, \\
G_{\mathcal{S}(\ell) \rightarrow \mathcal{C}(j) \mid \mathcal{P}} & =G_{\mathcal{S}(\ell) \rightarrow \mathcal{C}(j) \mid \mathcal{I}_{t}}, \\
\operatorname{son}\{\mathcal{S}(\ell)\} & =\operatorname{son}\{\mathcal{S}(\ell)\} \cup\{\mathcal{C}(j)\}, \\
\operatorname{pa}\{\mathcal{C}(j)\} & =\operatorname{pa}\{\mathcal{C}(j)\} \cup\{\mathcal{S}(\ell)\} .
\end{aligned}
$$

(b) Set $\mathcal{I}_{t+1}=\cup_{k=1}^{\left|\mathcal{I}_{t}\right|} \mathcal{S}_{\mathcal{I}_{t}(k)}, \mathcal{M}_{t+1}=\mathcal{M}_{t} \backslash \mathcal{I}_{t+1}$ and $t=t+1$.

\section{NUMERICAL SIMULATIONS}

In this section, we validate the proposed approach by means of synthetic signals, generated using a grid of interconnected elements running the FitzHugh-Nagumo model to simulate the behaviour of heart tissue [17], and contaminated by additive white Gaussian noise (AWGN) at a given signal to noise ratio (SNR). The Granger causal connectivity toolbox (see [18]) was used to obtain the basic pairwise and conditional causality relationships. A flat propagation wavefront is generated and a catheter with 9 sensors is placed inside the grid, with the wavefront entering it through the eighth sensor and exiting through the third one. The three approaches described in the paper (pairwise causality, full conditional causality and the novel hierarchical causality approach) are then applied.

Fig. 1 (left) shows an example of the networks obtained (using $M=11, p=0.01$ and SNR $=30 \mathrm{~dB}$ ). As expected, the pairwise approach includes a huge number of edges, since it cannot distinguish direct and indirect causal relationships. The full conditional approach does a much better job, but still provides too many cross-connections across the nodes. Finally, the hierarchical scheme includes less cross-connections and has a lower computational cost. The effect of the different factors (the number of lags, $M$, and the $p$-value) is shown in Fig. 1 (center and right), whereas Table 1 shows the effect of changing the SNR. ${ }^{4}$ On the one hand, the pairwise technique provides very good results in terms of sensitivity (detecting all the edges), but very poor results in terms of specificity (introducing many false edges). On the other hand, the full conditional scheme obtains very good results in terms of specificity, but not so good sensitivity results. Finally, the hierarchical approach provides the best sensitivity results, performing well also in ţrms of specificity and accuract

In this paper, we have introduced a novel hierarchical approach to infer Granger causality relationships among multi-

\footnotetext{
${ }^{4}$ Figures of merit used: accuracy $\left(\frac{T P+T N}{P+N}\right)$, sensitivity $\left(\frac{T P}{P}\right)$ and specificity $\left(\frac{T N}{N}\right), P$ denotes the number of positive instances (i.e., existing edges), $N$ the number of negative instances, $T P$ the number of correctly detected existing edges and $T N$ the number of correctly detected missing edges.
} 
Table 1. Performance vs. SNR ( $M=11, p=0.01)$

\begin{tabular}{|l|c|c|c|c|c|c|}
\cline { 2 - 7 } \multicolumn{1}{c|}{} & \multicolumn{3}{c|}{ Hierarchical } & \multicolumn{3}{c|}{ Full Cond. } \\
\hline SNR & Acc. & Sen. & Spe. & Acc. & Sen. & Spe. \\
\hline $10 \mathrm{~dB}$ & 0.860 & 0.516 & 0.903 & 0.892 & 0.380 & 0.956 \\
$15 \mathrm{~dB}$ & 0.850 & 0.591 & 0.882 & 0.889 & 0.520 & 0.935 \\
$20 \mathrm{~dB}$ & 0.846 & 0.681 & 0.866 & 0.886 & 0.677 & 0.912 \\
\hline
\end{tabular}

channel intra-cardiac electrocardiograms. The proposed scheme avoids detecting indirect causal links (as in pairwise approaches), and has a similar performance and lower computational cost than the full conditional causality method.

\section{REFERENCES}

[1] William B. Kannel and Emelia J. Benjamin, "Status of the epidemiology of atrial fibrillation," Medical Clinics of North America, vol. 92, no. 1, pp. 17-40, 2008.

[2] Concepción Moro Serrano and Antonio HernándezMadrid, "Atrial fibrillation: Is it an epidemic?," Revista Española de Cardiología, vol. 62, no. 1, pp. 10-14, 2009.

[3] Stanley Nattel, Duosheng Li, and Lixia Yue, "Basic mechanisms of atrial fibrillation-very new insights into very old ideas," Annual Review of Physiology, vol. 62, no. 1, pp. 51-77, January 2000.

[4] Stanley Nattel, Akiko Shiroshita-Takeshita, Bianca J.J.M. Brundel, and Léna Rivard, "Mechanisms of atrial fibrillation: Lessons from animal models," Progress in Cardiovascular Diseases, vol. 48, no. 1, pp. 9-28, July/August 2005.

[5] David E. Krummen and Sanjiv M. Narayan, "Mechanisms for the initiation of human atrial fibrillation," Heart Rhythm, vol. 6, no. 8, Supplement, pp. S12-S16, August 2009.

[6] Luca Faes, Alberto Porta, and Giandomenico Nollo, "Testing frequency-domain causality in multivariate time series," Biomedical Engineering, IEEE Transactions on, vol. 57, no. 8, pp. 1897-1906, 2010.

[7] Samantha Kleinberg and George Hripcsak, "A review of causal inference for biomedical informatics," Journal of biomedical informatics, vol. 44, no. 6, pp. 1102-1112, 2011.

[8] Steven L. Bressler and Anil K. Seth, "Wiener-Granger causality: a well established methodology," NeuroImage, vol. 58, no. 2, pp. 323-329, 2011.
[9] L Faes, G Nollo, et al., "Assessing frequency domain causality in cardiovascular time series with instantaneous interactions," Methods of information in medicine, vol. 49, no. 5, pp. 453, 2010.

[10] Alberto Porta, Tito Bassani, Vlasta Bari, and Stefano Guzzetti, "Granger causality in cardiovascular variability series: Comparison between model-based and model-free approaches," in Engineering in Medicine and Biology Society (EMBC), 2012 Annual International Conference of the IEEE. IEEE, 2012, pp. 3684 3687.

[11] Ulrike Richter, Luca Faes, Alessandro Cristoforetti, Michela Masè, Flavia Ravelli, Martin Stridh, and Leif Sörnmo, "A novel approach to propagation pattern analysis in intracardiac atrial fibrillation signals," Annals of biomedical engineering, vol. 39, no. 1, pp. 310-323, 2011.

[12] M Rodrigo, Alejandro Liberos, MS Guillem, J Millet, and Andreu M Climent, "Causality relation map: a novel methodology for the identification of hierarchical fibrillatory processes," in Computing in Cardiology, 2011. IEEE, 2011, pp. 173-176.

[13] Miguel Rodrigo, Maria S Guillem, Alejandro Liberos, José Millet, Omer Berenfeld, and Andreu M Climent, "Identification of fibrillatory sources by measuring causal relationships," in Computing in Cardiology (CinC), 2012. IEEE, 2012, pp. 705-708.

[14] Clive W. J. Granger, "Investigating causal relations by econometric models and cross-spectral methods," Econometrica, vol. 37, pp. 424-438, 1969.

[15] Petre Stoica and Yngve Selen, "Model-order selection: a review of information criterion rules," Signal Processing Magazine, IEEE, vol. 21, no. 4, pp. 36-47, 2004.

[16] J. Geweke, "Measures of conditional linear dependence and feedback between time series," Journal of the American Statistical Association, vol. 79, pp. 907-915, 1984.

[17] James P Keener and James Sneyd, Mathematical physiology, vol. 1, Springer, 1998.

[18] Anil K Seth, "A matlab toolbox for granger causal connectivity analysis," Journal of neuroscience methods, vol. 186, no. 2, pp. 262-273, 2010. 